\title{
Role of rapid antibody and ELISA tests in the evaluation of serological response in patients with SARS-CoV-2 PCR positivity
}

\author{
Yasemin Cosgun ${ }^{1}$ - Ayse Basak Altas ${ }^{1} \cdot$ Esra Akkan Kuzucu $^{1} \cdot$ Rahmet Guner $^{2} \cdot$ Sebnem Erdinc $^{3} \cdot$ Fatma Eser $^{2}$. \\ Esra Kaya Kilic ${ }^{3}$. Gulay Korukluoglu ${ }^{1}$
}

Received: 8 September 2020 / Accepted: 8 March 2021 / Published online: 7 April 2021

(C) Institute of Microbiology, Academy of Sciences of the Czech Republic, v.v.i. 2021

\begin{abstract}
From 7 to 8 days after the onset of symptoms in COVID-19 infection, the sensitivity of serological tests was found to be higher than that of nucleic acid tests. The aims of this study were to investigate antibody levels in patients with SARS-CoV-2 infection, to examine the relationship between antibody levels and virus load, and to evaluate the performance of 2 different commercial kits. A total of 103 patients with confirmed SARS-CoV-2 infection were included in the study. Antibodies against SARS-CoV-2 in serum samples taken from patients were investigated simultaneously with anti-SARS-CoV-2 IgG and IgA ELISAs (Euroimmun) and COVID-19 (SARS-CoV-2) IgG/IgM (Deep Blue) kits. No positivity was detected with any of the test kits in $18(17.4 \%)$ of the 103 samples. In symptomatic patients, $100 \%$ of IgM and IgA tests were found to be positive in the group sampled after 10 days, while $100 \%$ of $\mathrm{IgG}$ tests were found positive after 20 days. The sensitivity of the Deep Blue COVID-19 IgG antibody kit was calculated as $81.48 \%$ and the specificity was $97.96 \%$. While there was no statistically significant difference between the PCR CT and ELISA OD values, a positive correlation was found between the ELISA OD values and the days since the date of symptom initiation. The sensitivity and specificity of the rapid antibody test used in this study were found to be quite high. In conditions where ELISA tests cannot be applied, it is thought that it can give an idea in terms of the presence of antibodies as a simple and fast test. Although ELISA tests are valuable in the diagnosis of COVID-19 during the acute period, they are tests that can be used safely in the diagnosis of previous infections and seroepidemiological studies.
\end{abstract}

\section{Introduction}

A new coronavirus emerged in China in December 2019, causing the acute respiratory infection now known as coronavirus disease 2019 (COVID-19). The virus has been identified as a betacoronavirus associated with SARS coronavirus (SARS-CoV) and was therefore named SARS-CoV-2 (https://www.who.int/emergencies/diseases/novel-

Yasemin Cosgun

yasemincosgun2006@yahoo.com.tr

1 General Directorate of Public Health, Department of Microbiology Reference and Biological Products Laboratories, Ministry of Health, Ankara, Turkey

2 Department of Infectious Disease and Clinical Microbiology, Yildirim Beyazit University, Ankara City Hospital, Ankara, Turkey

3 Department of Infectious Diseases and Clinical Microbiology, Ankara Training and Research Hospital, Ankara, Turkey coronavirus-2019). Rapid identification of the virus and sharing of its genetic sequence enabled the rapid availability of real-time polymerase chain reaction (PCR) diagnostic tests that support case detection and monitoring. These tests have helped to identify patients and aided in efforts to limit the spread of the virus. Although serological tests are fast, easy to apply, and inexpensive, they may not detect the presence of infection in the acute phase of the disease, as a certain period of time must pass for antibody formation after the virus is encountered. Since false negative results may be seen in this period, it may be more appropriate to use PCR tests (To et al. 2020). However, the success of PCR tests in nasopharyngeal swab samples has been reported to be around 54-74\%. It has been reported that this can be caused by a variety of reasons, such as the uptake technique, time of sampling, material used, sample transfer, and low viral load due to suppression by host immunity (Yang et al. 2020). From 7 to 8 days after the onset of symptoms, the sensitivity of serological tests was found to be greater than that of nucleic acid tests (Zhao et al. 2020; Guo et al. 2020). Moreover, $100 \%$ seropositivity was reported 
in patients 10 days after the onset of symptoms (Lassaunière et al. 2020). This increases the value of serological tests in acute diagnosis, especially in patients with mild progress and late admission to the hospital. Serological tests are also very important for contact tracing, determination of virus carriers, and epidemiological studies. During an epidemic, one of the main challenges is identifying asymptomatic infection. These individuals may be the main source of infection, as they do not show any distinguishable symptoms (Long et al. 2020). Serological tests can detect mildly infected individuals and are important for determining the extent of disease spread (Okba et al. 2020). With epidemiological studies, it is possible to determine the disease burden, reveal the rate of asymptomatic infection, acquire information about the course of the disease, and determine the spread rate of the virus in places such as families, society, nursing homes, nurseries, and military units, thus making a significant contribution to taking control measures. Serological tests are also needed in terms of evaluating the results of vaccine trials and determining therapeutic plasma donors (Amanat et al. 2020). In addition, studies in this area are needed to determine which antibody target area and which commercial kits give more accurate and reliable results (Okba et al. 2020). In addition, point-of-care tests (POCT) and enzyme-linked immunoassay (ELISA) tests have come to the forefront in the investigation of antibody response (Elslande et al. 2020; Lippi et al. 2020). These tests also have advantages and disadvantages. Differences such as cross reactivity and the need for evaluation by an experienced healthcare professional can place limitations on the tests. In addition, there may be large differences between the infected individuals, due to the genetic characteristics of the individual, viral load, or other reasons that are not yet known (Zhao et al. 2020; Long et al. 2020). The aims of this study were to investigate antibody levels in patients with SARS-CoV-2 infection, evaluate the relationship between antibodies and viral load, and compare the performance of the 2 commercial kits.

\section{Material and methods}

\section{Sample selection}

A total of 103 patients who had SARS-CoV-2 infection and whose diagnosis was confirmed by nucleic acid tests were included in the study. Of the 103 patients, 92 were symptomatic and 11 were asymptomatic. All of the symptomatic patients were hospitalized but did not need intensive care, and the asymptomatic group consisted of the contact relatives of these patients. Serum samples were heat-inactivated at $56{ }^{\circ} \mathrm{C}$ for $60 \mathrm{~min}$, aliquoted, stored at $4{ }^{\circ} \mathrm{C}$, and used within 5 days. Prior to the study, all of the samples were brought to room temperature and centrifuged for a short time. The presence of immunoglobulin $\mathrm{A}(\operatorname{Ig} \mathrm{A}), \operatorname{IgM}$, and IgG antibodies against SARS-CoV-2 was investigated simultaneously in serum samples taken from the patients. At the time of blood sampling, information about symptom onset, date of PCR positivity, age, gender, and presence of symptoms were recorded.

\section{Serological tests}

\section{Rapid antibody test}

COVID-19 (SARS-CoV-2) IgG/IgM antibody tests (Colloidal Gold-Deep Blue/China-Germany, No.IFU-COVIDIgG/ IgM-01.Ver.1.3) were performed according to the manufacturer's instructions. The test method used was the immunochromatographic lateral flow assay. Added to the specimen well on the individual test cassettes was $10 \mu \mathrm{L}$ serum, followed by the addition of the supplied buffer. The result was read visually after $10 \mathrm{~min}$. Signals were considered positive.

\section{Enzyme-linked immunosorbent assay}

The anti-SARS-CoV-2 IgG and IgA ELISAs (Euroimmun Medizinische Labordiagnostika, Lübeck, Germany; Cat \# EI 2606-9601 G and EI 2606-9601 A) were performed according to the manufacturer's instructions. In 2 separate semiquantitative ELISAs, either IgA or IgG antibodies against SARS-CoV-2 spike protein subunit 1 (S1) are detected in human serum. According to the manufacturer's recommendations, $<0.8$ was considered negative, $\geq 0.8$ and $<1.1$ were considered borderline, and $\geq 1.1$ was considered positive. However, for sensitivity and specificity, 1.1 was used as a more stringent cut-off value for positive results and all values $<1.1$ were considered negative. Sensitivity and specificity of the rapid antibody kit were calculated according to the ELISA kit.

\section{Statistical analysis}

IBM SPSS Statistics for Windows 20.0 (IBM Corp., Armonk, NY, USA) was used to evaluate the data obtained from the study. The chi-square test was used to compare categorical variables, and coherence (\% agreement) was determined by calculating the Cohen kappa value. The Pearson correlation coefficient was used to examine the relationships between the variables and the relationships were also shown as a scatter plot. Statistical significance was accepted as $p<0.05$ in all of the statistical analyses. 
Fig. 1 Positivity detection rates (\%) of the kits according to the complaint initiation dates

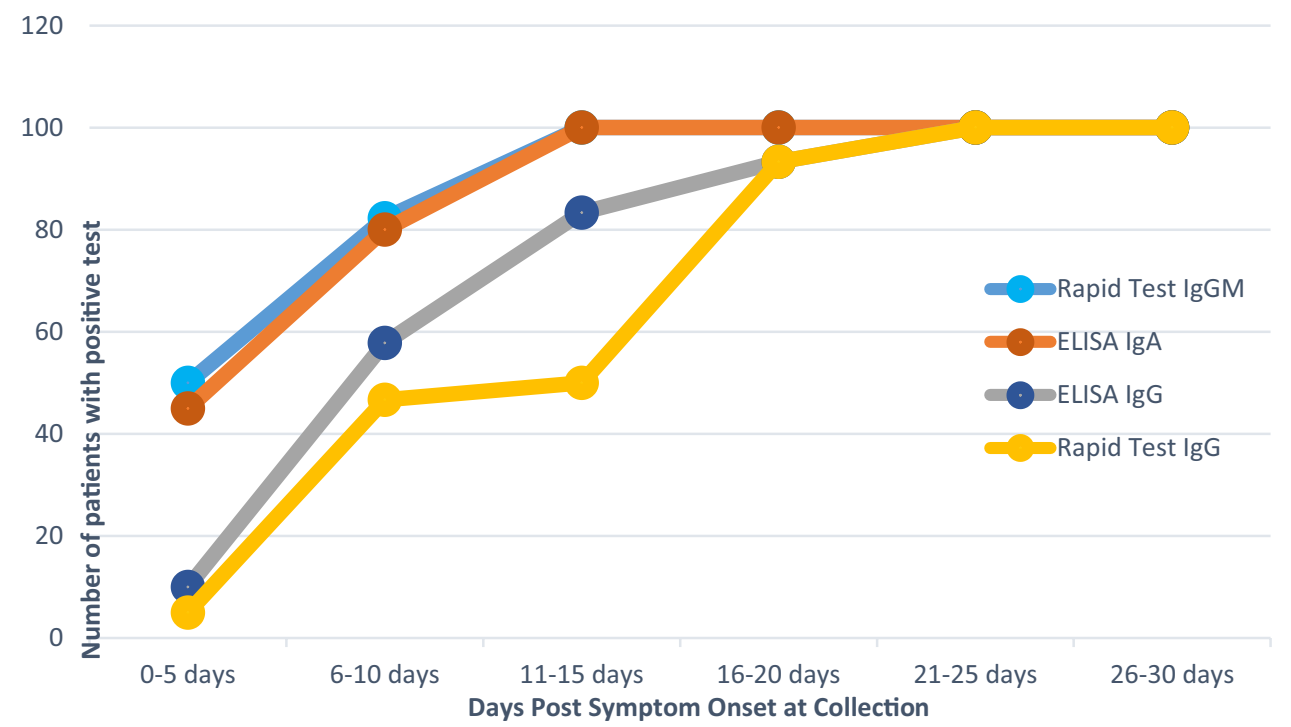

\section{Results}

The cases included in this study consisted of 49 female $(47.5 \%)$ and 54 male $(52.5 \%)$ patients.

When evaluated according to the date of complaint in the symptomatic patients, $100 \%$ of the $\operatorname{IgM}$ and $\operatorname{IgA}$ tests were found to be positive in the group sampled after day 10 , while $100 \%$ of the IgG tests were found to be positive in the group sampled after day 20 . While approximately half of the $\operatorname{IgM}$ and $\operatorname{IgA}$ tests were found positive in the patient blood samples taken in the first 5 days, only $10 \%$ of the $\operatorname{IgG}$ tests were found to be positive. Between days 5 and 10, it was found that $80 \%$ of the $\operatorname{IgM}$ and $\operatorname{IgA}$ tests were positive, as were $57 \%$ of the IgG tests; between days 11 and 15 , it was found that $100 \%$ of the IgM and IgA tests were positive, as were $83 \%$ of the IgG tests; between days 16 and 20 , it was found that $93 \%$ of the $\mathrm{IgG}$ test were positive; and on day 21 , it was found that $100 \%$ of the $\operatorname{IgG}$ tests were positive. No positivity was detected with any of the test kits in $18(17.4 \%)$ of the 103 samples. The rate of not detecting antibodies on any test was $12 \%(11 / 92 \%)$ in the symptomatic patients, but they were all samples taken on day 10 or before. In other words, a positive result was obtained with any of the tests in all of the symptomatic patients sampled after day 10. Antibodies were detected in only 4 (36.3\%) of 11 asymptomatic patients, and all 4 of these patients were sampled after day 10. Of the 7 patients without antibodies, 4 were sampled 10 days before the onset of symptoms and 3 were sampled 10 days after (Fig. 1). The specificity and sensitivity of the Deep Blue COVID-19 IgG antibody kit when compared with the Euroimmun anti-SARS CoV-2 IgG kit were calculated. The sensitivity was $81.48 \%$, while the specificity was $97.96 \%$ (Table 1). Borderline results were considered negative (Table 1).

\section{Consistency between serological tests}

To evaluate the agreement between the ELISA and POCT, the same result detection rates and kappa values were calculated with both kits. Agreement between the Deep Blue COVID-19 IgG and Euroimmun IgG ELISA was calculated as $89.3 \%$ (kappa value $=0.73$ ). Agreement between the Deep Blue COVID-19 IgM and Euroimmun IgA ELISA was calculated as $80.5 \%$ (kappa value $=0.51$ ). A weak negative correlation was found between the PCR test $\mathrm{Ct}$ values and ELISA IgA and IgG OD index values of the patients, but it was not statistically significant (Figs. 2 and 3).

Table 1 Specificity and sensitivity of the Deep Blue COVID-19 IgG antibody kit when compared with the Euroimmun anti-SARS-CoV-2 IgG kit

\begin{tabular}{lllllll}
\hline Positive/total & Sensitivity (95\% CI) & Negative/total & Specificity (95\% CI) & PPV (95\% CI) & NPV (95\% CI) & Accuracy (95\% CI) \\
\hline $44 / 54$ & $81.48 \%(68.57-$ & $48 / 49$ & $97.96 \%(89.15-$ & $97,78 \%(86.3-$ & $82.76 \%(73.26-$ & $89.32 \%(81.69-94.55)$ \\
& $90.75)$ & & $99.95)$ & $99.68 \%)$ & $89.37)$ & \\
\hline
\end{tabular}

$P P V$ positive predictive value, $N P V$ negative predictive value, $C I$ confidence of interval 
Fig. 2 Correlation between PCR $\mathrm{Ct}$ values and anti-SARS-CoV-2 $\mathrm{Ig} A$ OD index. The value of $\mathrm{R}$ is $-0.043, p=0.717$

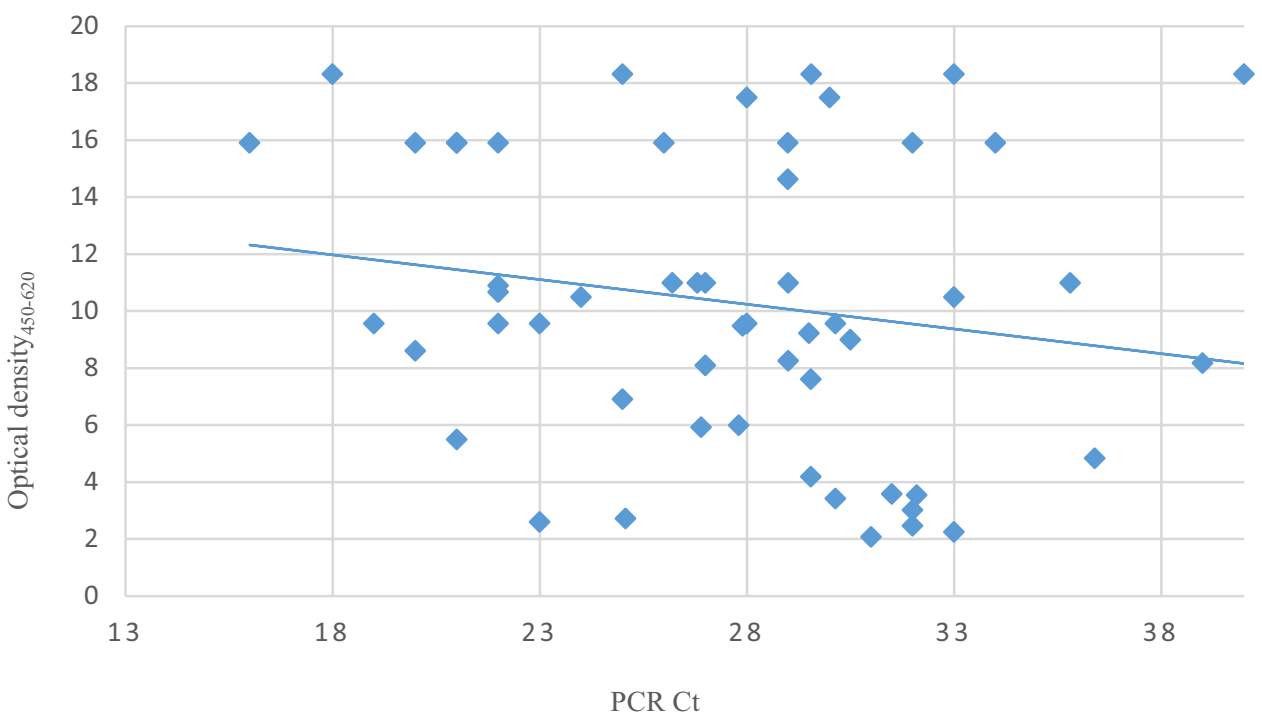

A positive and statistically significant correlation was found between the symptom onset dates and the $\operatorname{IgA} / \operatorname{IgG}$ levels (Figs. 4 and 5).

A positive and statistically significant correlation was found between the number of days since PCR positivity and the $\mathrm{IgA} / \mathrm{IgG}$ levels (Fig. 6 and 7).

In these calculations, no statistically significant difference was found between taking the symptom onset dates or the PCR positivity dates as the beginning.

\section{Discussion}

There may be various reasons for the absence of antibody positivity with any of the kits tested in $18(17.4 \%)$ of the 103 patients who had the infection in the current study. It was thought that the main reason might have been due to insufficient time after infection for antibody formation. Antibodies were not detected in $11(12 \%)$ of 92 of the symptomatic patients, all of whom were sampled in the first 10 days. In this study, antibodies were detected in all of the symptomatic patients who were sampled after day 10 . According to these rates, the rate of antibody development against SARS-CoV-2 seemed to be quite high in the symptomatic patients. Elslande et al. (2020) detected $89.5 \%$ IgG positivity using ELISA in their patient group with samples taken between days 14 and 25. Zhao et al. (2020) found the seroconversion rate to be $93.1 \%$ in their study with 173 patients. While the presence of antibodies was $<40 \%$ among patients in the first 7 days of the disease, it rapidly increased to $100 \%$ after day 15 . Wölfel et al. (2020) detected seroconversion in $50 \%$ of patients on day 7 of the disease and found it in all of the patients on day 14. These rates are closely related to the severity of the disease and the time of
Fig. 3 Correlation between PCR $\mathrm{Ct}$ values and anti-SARS-CoV-2 IgG OD index. The value of $R$ is $-0.249, p=0.069$

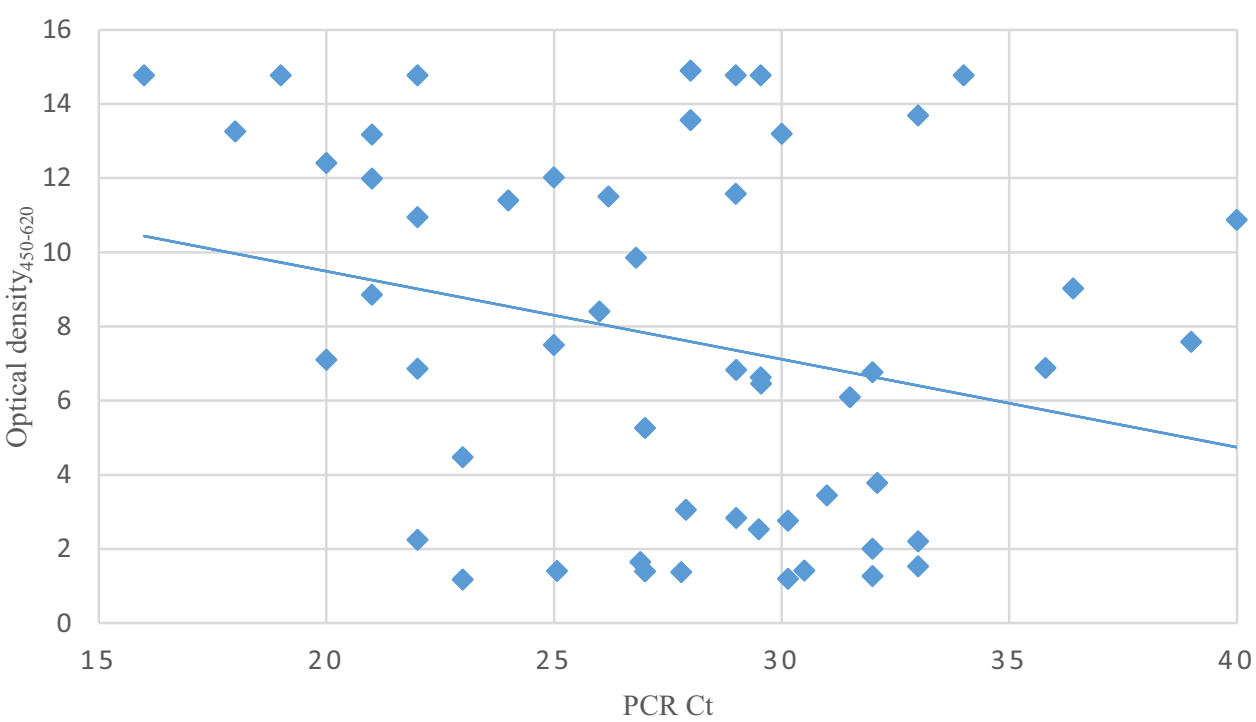


Fig. 4 Correlation between time (days) after symptom onset and anti-SARS-CoV-2 IgA OD index. The value of $R$ is 0.4165 , $p=0.0003$

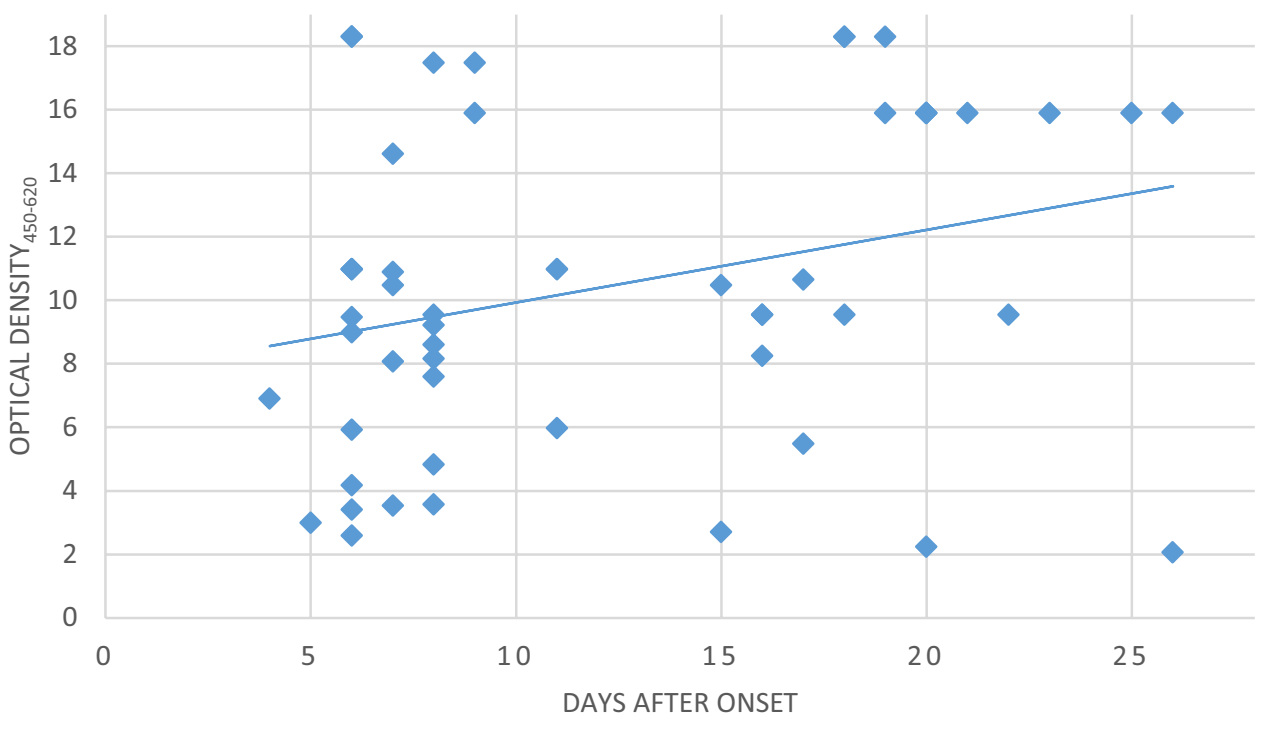

sample collection, as well as the antibody response generated by the patients. In the current study, the rate of antibody detection by any of the tests in the asymptomatic patients was $36.3 \%$ (4/11) and all of the samples were taken after day 10. Of the 7 patients with no antibodies, 4 had samples taken before day 10 and 3 had samples taken after day 10. It was observed that there were problems with detectable antibody formation in patients who had the disease asymptomatically. However, these patients were the ones who encountered the virus and had positive PCR tests. When they encountered the virus again, an immune memory may have formed in their bodies that created a strong and detectable antibody response and prevented them from re-infection. Long et al. (2020) identified $6.1 \%$ asymptomatic patients with a serological response in their serological study in a group of 164 patients. Chen et al. (2020) found that PCR and tests were negative in 105 healthcare workers who were exposed to 4 laboratory-confirmed patients, while they found seropositivity in $17.14 \%(18 / 105)$ of them with enzyme immunoassay and microneutralization tests. They reported that the serological tests were useful in identifying asymptomatic or subclinical infection of SARSCoV-2 in individuals who were in close contact with COVID-19 patients. Yongchen et al. (2020), in a study they conducted on 21 diseases, detected antibodies in only 1 of 5 asymptomatic patients, while antibodies were detected in all of the symptomatic patients with severe and mild prognosis. In the current study, when the positive patients were classified according to the time that had elapsed between the onset of complaints and the date of blood collection, about half of the IgM and IgA tests were found to be positive in patient blood samples taken in the first 5 days, while
Fig. 5 Correlation between time (days) after symptom onset and anti-SARS-CoV-2 IgG OD index. The value of $R$ is 0.3605 , $p=0.008$

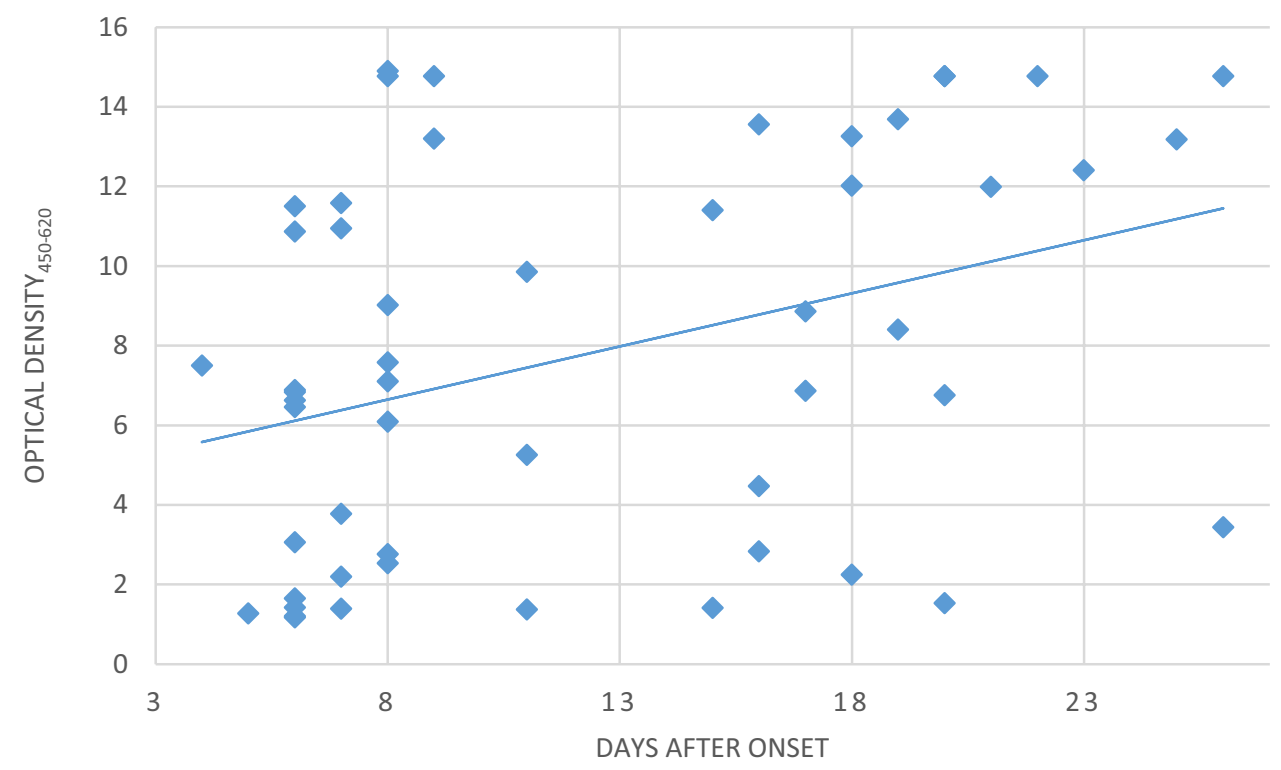


Fig. 6 Correlation between time (days) after PCR positive date and anti-SARS-CoV-2 IgA OD index. The value of $R$ is 0.3935 , $p=0.0005$

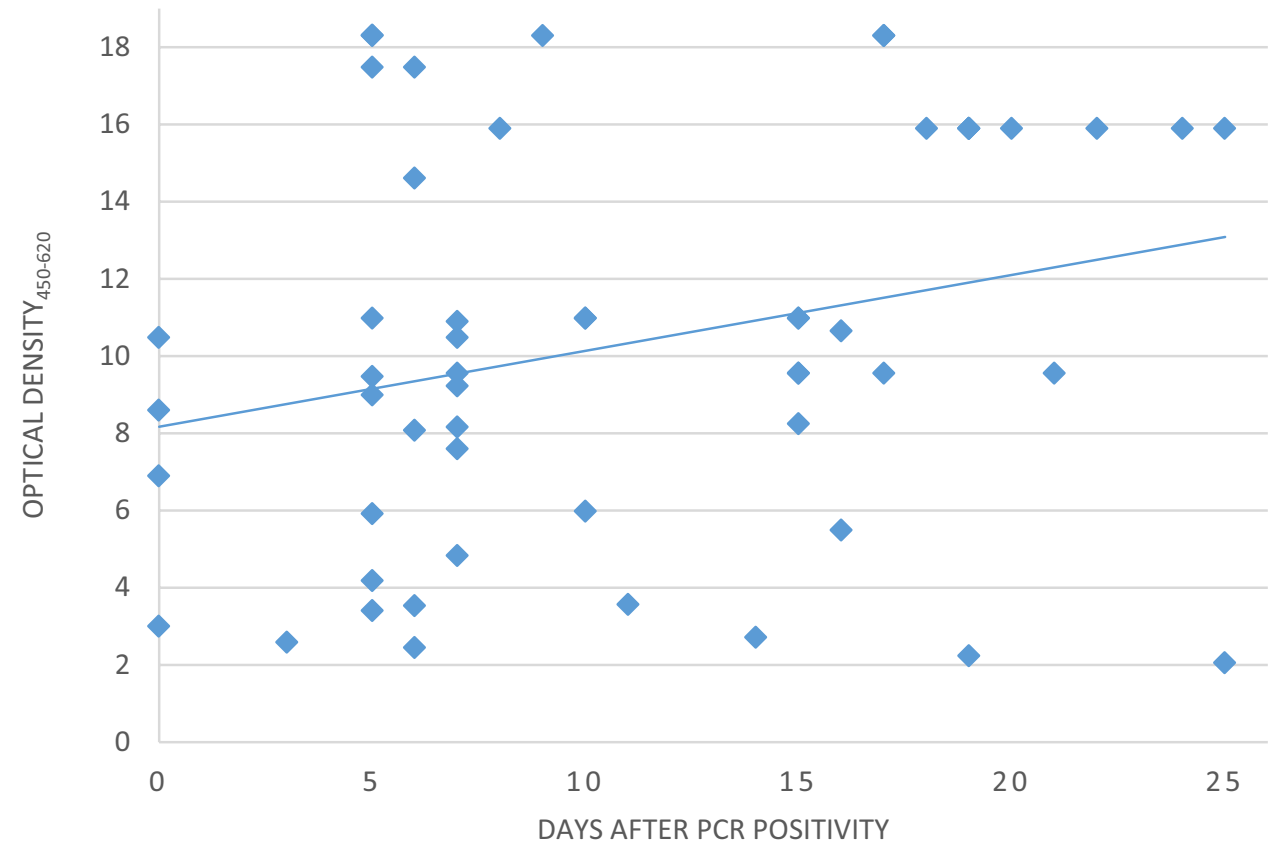

only $10 \%$ of the IgG tests were found to be positive. Fierz and Walz (2020) reported that it may be more appropriate to use PCR tests, as negative results can be seen with serological tests, especially in the first 10 days of infection. However, according to the results of the current study, antibodies were detected in $50 \%$ of the patients with serological tests, even in the first 5 days, and in $80 \%$ of the patients in the between days 5 and 10 . Thus, it seemed possible to detect patients using serological tests who were not able to be detected using the PCR test and diagnose acute infection. In the symptomatic patients, between days 5 and 10, it was found that $80 \%$ of the $\operatorname{IgM}$ and $\operatorname{IgA}$ tests were positive, as were $57 \%$ of the IgG tests; between days 11 and 15 , it was found that $100 \%$ of the $\operatorname{IgM}$ and $\operatorname{Ig}$ A tests were positive, as were $83 \%$ of the $\operatorname{IgG}$ tests; between days 16 and 20 , it was found that $93 \%$ of the $\mathrm{IgG}$ test were positive; and after day 201 , it was found that $100 \%$ of the IgG tests were positive. Zhang et al. (2020) found $50 \%$ and $81 \%$ IgM and IgG positivity on day 0 (first sampling day), which increased to $81 \%$ and $100 \%$, respectively, on day 5 . Therefore, COVID-19 cannot be excluded at an early stage when the viral serological test is negative. Antibody responses may be different among infected individuals (Long et al. 2020). Okba et al. (2020) reported that $\operatorname{IgG}$ and $\operatorname{IgA}$ kinetics vary between patients with different disease severity. In the current study, while $100 \%$ of the $\operatorname{IgM}$ and $\operatorname{IgA}$ tests were found to be
Fig. 7 Correlation between time (days) after PCR positive date and anti-SARS-CoV-2 IgG OD index. The value of $R$ is 0.3381 , $p=0.0132$

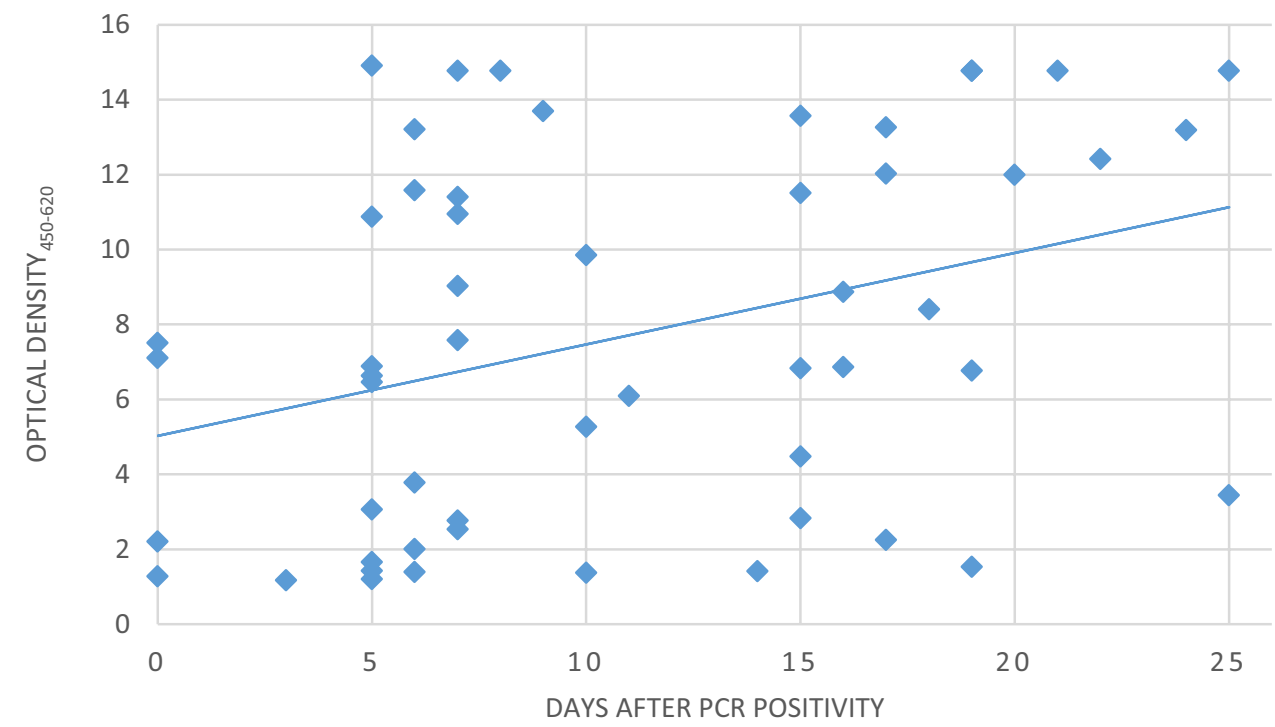


positive in the group sampled after day 10 , it was found that $100 \%$ of the IgG tests were positive in the group sampled after day 20 (Fig. 1). Lassaunière et al. (2020) reported $100 \%$ seropositivity in patients 10 days after the onset of symptoms, but all of the patients were adults with severe clinical conditions. In this study, a similar result was obtained in the symptomatic group. Guo et al. (2020) detected $85.4 \%$ anti-SARS-CoV-2 IgM and $92.7 \%$ IgA antibodies in plasma samples collected within $0-7$ days. They determined the median day for detection of IgM and $\operatorname{Ig} \mathrm{A}$ antibodies in these acute phase samples as day 5. Additionally, $77.9 \%$ anti-SARS-CoV-2 IgG antibody positivity was found in samples collected between days 10 and 18. Herein, 83.3\% ELISA IGG positivity was detected in samples collected between days 11 and 15. Deeks et al. (2020) examined 57 published articles and calculated the sensitivity of antibody tests as $30.1 \%$ between days 1 and $7,72.2 \%$ between days 8 and 14, and $91.4 \%$ between days 15 and 21 . They reported that these sensitivity rates were too low to have a primary role in the diagnosis of COVID-19 in the first week of the disease, but could have a complementary role in those who applied later when the PCR tests were negative. When the consistency of the results between the kits was examined in the current study, agreement between the Deep Blue COVID-19 IgG and Euroimmun IgG ELISA was calculated as $89.3 \%$ (kappa value: 0.78 ). Inconsistency was determined in the result of only 11 samples, 10 of which were ELISA IgG-positive, rapid test-negative, and in 6 , the ELISA IgG OD index was found to be below 2. It was thought that the antibody level in the samples with a low OD index might not have formed a visible band with the rapid test. For the remaining 4 samples and also 1 sample detected as positive using the rapid test and negative using ELISA, it was thought that the target regions of the tests were composed of different antigenic structures or it could have been caused by other reasons that are not yet known. Nicol et al. (2020) reported that the agreement between lateral flow immunoassay was $97 \%(k=0.936)$ when compared with automated serological systems. In the current study, when the specificity and sensitivity of the Deep Blue COVID-19 IgG rapid antibody kit when compared with the Euroimmun anti-SARS-CoV-2 IgG kit were calculated, the sensitivity was calculated as $81.48 \%$ and specificity as $97.96 \%$. It was thought to perform quite well for a bedside rapid antibody test. Elslande et al. (2020) evaluated the diagnostic performance of 7 different rapid $\mathrm{IgG} / \mathrm{IgM}$ antibody tests in samples collected between days 14 and 25 and reported that the sensitivity of the tests for $\operatorname{IgG}$ was found to be between 0.192 .1 and $100 \%$, and the specificity was $\geq 90.3 \%$. Herein, agreement between the Deep Blue COVID-19 IgGM and Euroimmun IgA ELISA was calculated as $80.5 \%$ (kappa value: 0.51 ). Although the results of different kits and methods were found, compatibility between the $\operatorname{IgA}$ and $\operatorname{IgM}$ was found to be very low, suggesting that the measurement of these antibodies would not be interchangeable. While the IgA positivity was found to be $70.8 \%$ (73 patients), IgM positivity was found to be $74.7 \%$ (77 patients). There was no statistically significant difference between the 2 tests. However, when the 2 antibodies were used together, a sensitivity of $82.5 \%(85 / 103)$ was reached in the entire patient group. Therefore, it was believed that IgA levels can be very useful, especially in the diagnosis of the acute period, since the infection is one that starts in respiratory epithelial cells. Lippi et al. (2020) found that the sensitivity of $\operatorname{IgA}$ was higher than that of IgM. However, there may be differences between the sensitivity of the tests depending on many factors, such as the test methods used, target regions, and patient population. The advantage of rapid antibody tests is that they can be performed at the bedside. ELISA tests, on the other hand, are done in laboratories and require experienced staff and expert evaluation. In this respect, the ability to perform rapid antibody tests, especially with a kit that has a sensitivity of over $80 \%$, and the ability to test 1 drop of blood taken from a finger at home or at the patient's bedside can provide a great advantage. In the current study, a weak negative correlation was found between the PCR test $\mathrm{Ct}$ values and IgA and IgG OD index values of the patients, but it was not statistically significant (Figs. 2 and 3). Jin et al. (2020) showed that in patients they followed up from the onset of symptoms until the day 55, the antibody levels increased as the viral load decreased in the first 20 days, and then, the increase in antibody did not continue. As in many viral infections, it is seen that in COVID-19 infection, as the viral load decreases, the antibody response increases or, more accurately, the viral load in the body decreases as the antibody level increases depending on the immune response. In the current study, a positive and statistically significant correlation was found between the symptom onset dates or the number of days since PCR positivity and the $\mathrm{IgA} / \mathrm{IgG}$ levels (Figs. 4, 5, 6, and 7). This meant that the antibody levels increased as time passed over the illness and the viral load decreased. Although this result was an expected result, clearer information can be obtained with studies about how the antibody levels will be affected over longer periods. In a study by Zhao et al. (2020), although antibody levels were detected below $40 \%$ in the first 7 days, they reached $100 \%$ after day 15 . However, since our study included a group of patients who were sampled for a maximum of 26 days, the relationship between the time elapsed from the onset of the disease and antibody levels in the future could not be evaluated. Studies have found a good level of correlation between SARS-CoV-2 ELISA antibody titers and neutralizing antibody titers (Okba et al. 2020; Kohmer et al. 2020). In another study, good correlation was found between the Euroimmun ELISA IgG and plaque reduction neutralization 
(PRNT) results and it was reported that this could be an indicator of protective immunity. Perera et al. (2020) reported a correlation with the ELISA test developed against the receptor-binding domain of the spike protein of the virus, and with the microneutralization and PRNT tests, and it was shown that there was no cross reactivity. ELISA tests can also be used as a good serological test for laboratories where neutralizing antibody tests are not performed.

\section{Conclusion}

Although many different specificity and sensitivity values have been reported for rapid antibody tests, the sensitivity and specificity of the rapid antibody test used in the current study were found to be quite high. In conditions where ELISA tests cannot be applied, it is thought that it can give an idea in terms of the presence of antibodies as a simple and fast test. Although ELISA tests are valuable in the diagnosis of COVID-19 during the acute period, they are tests that can be used safely in the diagnosis of previous infections and seroepidemiological studies. It is an important result of the current study that much more reliable results can be obtained in blood samples taken after day 10 in the detection of IgM and $\operatorname{IgA}$ antibodies, and after day 20 in the detection of IgG. The results of serological studies also increase prevention in terms of creating basic results for plasma therapy, monoclonal antibody therapies, or vaccine studies.

\section{References}

Amanat F, Stadlbauer D, Strohmeier S et al (2020) A serological assay to detect SARS-CoV-2 seroconversion in humans. Nat Med 26:1033-1036. https://doi.org/10.1038/s41591-020-0913-5

Chen Y, Tong X, Wang J et al (2020) High SARS-CoV-2 antibody prevalence among healthcare workers exposed to COVID-19 patients. J Infect 81:420-426. https://doi.org/10.1016/j.jinf.2020.05.067

Deeks JJ, Dinnes J, Takwoingi Y et al (2020) Antibody tests for identification of current and past infection with SARS-CoV-2. Cochrane Database Syst Rev 6:CD013652. https://doi.org/10.1002/ 14651858.CD013652

Fierz W, Walz B (2020) Antibody dependent enhancement due to original antigenic sin and the development of SARS. Front Immunol 11:1120. https://doi.org/10.3389/fimmu.2020.01120

Guo L, Ren L, Yang S et al (2020) Profiling early humoral response to diagnose novel coronavirus disease (COVID-19). Clin Infect Dis 71:778-785. https://doi.org/10.1093/cid/ciaa310

Jin Y, Wang M, Zuo Z et al (2020) Diagnostic value and dynamic variance of serum antibody in coronavirus disease 2019. Int J Infect Dis 94:49-52. https://doi.org/10.1016/j.ijid.2020.03.065

Kohmer N, Westhaus S, Rühl C et al (2020) Clinical performance of different SARS-CoV-2 IgG antibody tests. J Med Virol 92:22432247. https://doi.org/10.1002/jmv.26145
Lassaunière R, Frische A, Harboe ZB, et al (2020) Evaluation of nine commercial SARS-CoV-2 immunoassays. medRxiv 20056325. https://doi.org/10.1101/2020.04.09.20056325

Lippi G, Salvagno GL, Pegoraro M et al (2020) Assessment of immune response to SARS-CoV-2 with fully automated MAGLUMI 2019-nCoV IgG and IgM chemiluminescence immunoassays. Clin Chem Lab Med 58:1156-1159. https://doi.org/10.1515/ cclm-2020-0473

Long Q, Deng H, Chen J, et al (2020) Antibody responses to SARSCoV-2 in COVID-19 patients: the perspective application of serological tests in clinical practice. medRxiv 20038018. https://doi. org/10.1101/2020.03.18.20038018

Nicol T, Lefeuvre C, Serri O et al (2020) Assessment of SARS-CoV-2 serological tests for the diagnosis of COVID-19 through the evaluation of three immunoassays: two automated immunoassays (Euroimmun and Abbott) and one rapid lateral flow immunoassay (NG Biotech). J Clin Virol 129:104511. https://doi.org/10.1016/j. jcv.2020.104511

Okba NMA, Müller MA, Li W et al (2020) Severe acute respiratory syndrome coronavirus 2-specific antibody responses in coronavirus disease patients. Emerg Infect Dis 26:1478-1488. https://doi. org/10.3201/eid2607.200841

Perera RA, Mok CK, Tsang OT et al (2020) Serological assays for severe acute respiratory syndrome coronavirus 2 (SARS-CoV-2). Euro Surveill 25:2000421. https://doi.org/10.2807/1560-7917. ES.2020.25.16.2000421

To KK, Tsang OT, Leung WS et al (2020) Temporal profiles of viral load in posterior oropharyngeal saliva samples and serum antibody responses during infection by SARS-CoV-2: an observational cohort study. Lancet Infect Dis 20:565-574

Van Elslande J, Houben E, Depypere M et al (2020) Diagnostic performance of seven rapid IgG/IgM antibody tests and the Euroimmun IgA/IgG ELISA in COVID-19 patients. Clin Microbiol Infect 26:1082-1087. https://doi.org/10.1016/j.cmi.2020.05.023

Wölfel R, Corman VM, Guggemos W et al (2020) Virological assessment of hospitalized patients with COVID-2019. Nature 581:465469. https://doi.org/10.1038/s41586-020-2196-x

World Health Organization Press Conference. The World Health Organization (WHO) Has Oficially named the Disease Caused by the Novel Coronavirus as COVID-19. Available online: https:// www.who.int/emergencies/diseases/novel-coronavirus-2019

Yang Y, Yang M, Yuan J et al (2020) Comparative sensitivity of different respiratory specimen types for molecular diagnosis and monitoring of SARS-CoV-2 shedding. Innovation (NY) 1:100061. https://doi.org/10.1016/j.xinn.2020.100061

Yongchen Z, Shen H, Wang X et al (2020) Different longitudinal patterns of nucleic acid and serology testing results based on disease severity of COVID-19 patients. Emerg Microbes Infect 9:833836. https://doi.org/10.1080/22221751.2020.1756699

Zhang W, Du RH, Li B et al (2020) Molecular and serological investigation of 2019-nCoV infected patients: implication of multiple shedding routes. Emerg Microbes Infect 9:386-389. https://doi. org/10.1080/22221751.2020.1729071

Zhao J, Yuan Q, Wang H et al (2020) Antibody responses to SARSCoV-2 in patients with novel coronavirus disease 2019. Clin Infect Dis 71:2027-2034. https://doi.org/10.1093/cid/ciaa344

Publisher's Note Springer Nature remains neutral with regard to jurisdictional claims in published maps and institutional affiliations. 